\title{
Spontaneous Functional Recovery after Focal Damage in Neuronal Cultures
}

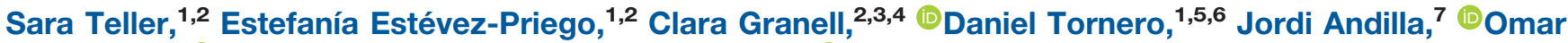 \\ E. Olarte, ${ }^{7,8}$ - Pablo Loza-Alvarez, ${ }^{7}$ Alex Arenas, ${ }^{9}$ and ${ }^{1}$ Jordi Soriano ${ }^{1,2}$
}

\author{
https://doi.org/10.1523/ENEURO.0254-19.2019
}

\begin{abstract}
${ }^{1}$ Departament de Física de la Matèria Condensada, Universitat de Barcelona, Barcelona 08028, Spain, ${ }^{2}$ Universitat de Barcelona Institute of Complex Systems (UBICS), Barcelona 08028, Spain, ${ }^{3}$ GOTHAM Lab-Institute for

Biocomputation and Physics of Complex Systems (BIFI), University of Zaragoza, Zaragoza 50018 , Spain, ${ }^{4}$ Department of Condensed Matter Physics, University of Zaragoza, Zaragoza 50009, Spain, ${ }^{5}$ Departament de Biomedicina, Facultat de Medicina, Institut de Neurociències, Universitat de Barcelona, Barcelona 08036, Spain, ${ }^{6} \mathrm{Centro}$ de Investigación Biomédica en Red sobre Enfermedades Neurodegenerativas (CIBERNED), ${ }^{7}$ ICFO-Institut de Ciències Fotòniques, The Barcelona Institute of Science and Technology, Castelldefels 08860, Spain, ${ }^{8}$ Universidad ECCI, Bogotá 111311, Colombia, and ${ }^{9}$ Departament d'Enginyeria Informàtica i Matemàtiques, Universitat Rovira i Virgili, Tarragona 43007, Spain
\end{abstract}

\begin{abstract}
Damage in biological neuronal networks triggers a complex functional reorganization whose mechanisms are still poorly understood. To delineate this reorganization process, here we investigate the functional alterations of in vitro rat cortical circuits following localized laser ablation. The analysis of the functional network configuration before and after ablation allowed us to quantify the extent of functional alterations and the characteristic spatial and temporal scales along recovery. We observed that damage precipitated a fast rerouting of information flow that restored network's communicability in about $15 \mathrm{~min}$. Functional restoration was led by the immediate neighbors around trauma but was orchestrated by the entire network. Our in vitro setup exposes the ability of neuronal circuits to articulate fast responses to acute damage, and may serve as a proxy to devise recovery strategies in actual brain circuits. Moreover, this biological setup can become a benchmark to empirically test network theories about the spontaneous recovery in dynamical networks.
\end{abstract}

Key words: calcium imaging; focal damage; functional recovery; laser microsurgery; network neuroscience; neuronal cultures

\section{Significance Statement}

Given the sheer size of the brain, in vitro models in the form of neuronal cultures have emerged as a promising tool to investigate dynamic and network alterations in detail on physical damage. Here we present a new experimental paradigm based on the combination of laser microsurgery and calcium fluorescence imaging to analyze network functional alterations after a focal lesion. We show that the network is not only able to cope with damage but that the regions around the lesion core actively participate in recovery, restoring the initial network activity levels in just $15 \mathrm{~min}$. Our approach offers interesting perspectives for modeling network functional loss and recovery in a number of damage actions, from stroke to degenerative disorders.

\section{Introduction}

The functional affectations in a neuronal circuit that arise from focal damage are complex. In the brain, the

Received June 27, 2019; accepted November 29, 2019; First published December 9, 2019.

The authors declare no competing financial interests. traumatic loss of neuronal tissue irreversibly disables the lesioned site and silences the connectivity pathways

Author contributions: S.T., J.A., P.L.-A., and J.S. designed research; S.T., J.A., and O.E.O. performed research; S.T., E.E.-P., C.G., D.T., and A.A. contributed unpublished reagents/analytic tools; S.T., E.E.-P., D.T., and J.S. analyzed data; S.T. and J.S. wrote the paper. 
emerging from and converging on it (Carrera and Tononi, 2014; Fornito et al., 2015). Damage leads to broad alterations in the spatiotemporal structure of neuronal dynamics that translate into functional deficits of diverse extend and severity (Alstott et al., 2009; Corbetta et al., 2015; Fornito et al., 2015; Siegel et al., 2016). The sudden activity loss, however, triggers substantial neuroplasticity, in which activity-dependent rewiring and strengthening drives functional reorganization and recovery (Murphy and Corbett, 2009; Zhu et al., 2010; van Meer et al., 2012), ultimately restoring partially or totally the altered brain functions.

Although the biological processes involved in damage and repair are well understood (Carmichael, 2016), the network mechanisms that facilitate swift response and functional recovery constitute a fundamental paradigm still to be completely understood (Majdandzic et al., 2013). These mechanisms are central to pinpoint the extent of affectation and evaluate the capacity of the circuit to restore function. Despite progress in vivo through animal models (Cheng et al., 2014; Lim et al., 2014), the sheer size and intricacy of brain circuits have fostered the development of in vitro approaches in which network damage and subsequent recovery can be examined in detail (Richard et al., 2010; Siddique and Thakor, 2014; Holloway and Gavins, 2016). At present, however, there are no dedicated in vitro models that can offer a high control on the delivered damage, large-scale network monitoring and detailed functional analysis of network's behavior.

To address these limitations, here we investigate the functional restoration of rat cortical networks in vitro on acute focal damage, delivered through highly focused ultrashort laser pulses that produce accurate laser ablation with micrometric resolution (Vogel et al., 2005; Thayil et al., 2008). The spontaneous activity of the cortical network is monitored through high-speed, whole-network calcium imaging (Orlandi et al., 2013; Teller et al., 2014, 2015), which allows us to quantify in detail the network

This research is part of MESO-BRAIN. The MESO-BRAIN Project has received funding from the European Union's Horizon 2020 Research and Innovation Programme Grant 713140 (to S.T., E.E.-P., J.A., O.E.O., P.L.-A., J.S.). J.S. and S.T. were supported by the Spanish Ministerio de Economia y Competitividad Projects FIS2013-41144-P, FIS2016-78507-C2-2-P, and FIS2017-90782-REDT (IBERSINC) and by the Generalitat de Catalunya Grant 2017-SGR-1061. C.G. was supported by Juan de la Cierva-Formación (Ministerio de Ciencia, Innovación y Universidades) and by the James S. McDonnell Foundation Postdoctoral Fellowship Grant 220020457. A.A. was supported by the Generalitat de Catalunya Project 2017-SGR-896, Spanish MINECO Projects FIS2015-71582-C2-1 and FIS2017-90782-REDT, ICREA Academia, and the James S. McDonnell Foundation Grant 220020325. J.A., O.E.O., and P.L.-A. were supported by the Spanish Ministerio de Economia y Competitividad (AEI/FEDER) Project FIS2016-80455-R, the "Severo Ochoa" Programme for Centers of Excellence in R\&D (SEV-2015-0522), Fundació Privada Cellex, Fundación Mig-Puig, Generalitat de Catalunya through the CERCA program, and Laserlab-Europe (EU-H2020 654148).

Correspondence should be addressed to Jordi Soriano at jordi.soriano@ub.edu.

https://doi.org/10.1523/ENEURO.0254-19.2019

Copyright @ 2020 Teller et al.

This is an open-access article distributed under the terms of the Creative Commons Attribution 4.0 International license, which permits unrestricted use, distribution and reproduction in any medium provided that the original work is properly attributed. functional alterations on damage and map the network interactions along recovery. We observed that damage precipitated a sudden fall of the global efficiency of the network, which gradually recovered to predamage levels in $\sim 15 \mathrm{~min}$. Recovery was mediated by an increased spontaneous activity of the regions around the lesion core, rerouting information flow to create new functional links or to strengthen existing ones. This rich plasticity evinces the capacity of the neuronal circuit to respond to damage as a global system, and hints at the existence of whole-network homeostatic mechanisms for circuit remodeling and functional restoration. To our knowledge, the study presented here is the first in vitro attempt to disclose the complexity of functional restoration on acute damage, and brings new opportunities to understand resilience and recovery in brain-like circuits from a networkbased perspective.

\section{Materials and Methods}

\section{Ethics statement}

All animal procedures were performed in accordance with the Ethical Committee for Animal Experimentation of the University of Barcelona, under order DMAH-5461.

\section{Experimental design \\ Clustered neuronal cultures}

Cortical neurons were dissected from Sprague-Dawley rat embryos at 18-19 d of development, following procedures previously described (Teller et al., 2014, 2015). Briefly, embryonic brains were dissected, cortical neurons dissociated by repeated pipetting, neurons suspended in an appropriate culture medium, and finally plated onto 13-mm glass coverslips (Marienfield-Superior) that incorporated four perforated circular cavities in a mold of polydimethylsiloxane (PDMS). Glasses and PDMS masks were attached together and autoclaved at $105^{\circ} \mathrm{C}$ for firm adhesion. PDMS cavities shaped minicultures that were 3 $\mathrm{mm}$ in diameter, $2 \mathrm{~mm}$ deep, and separated from one another by $1 \mathrm{~mm}$. The size of the minicultures was optimized to fit two of them in the field of view of the imaging system.

The absence of adhesive proteins in the glass substrate facilitated cell motility and aggregation, and ultimately shaped a network of dense neuronal islands (clusters) connected to one another (Segev et al., 2003; de SantosSierra et al., 2014; Teller et al., 2015). Neurons were seeded with a density of 2500 neurons $/ \mathrm{mm}^{2}$, providing $\sim 40$ clusters per culture, and were incubated in plating medium [Eagle's MEM (Invitrogen) supplemented with 5\% fetal calf serum (FCS; Invitrogen), $5 \%$ horse serum (HS; Invitrogen), $1 \mu \mathrm{l} / \mathrm{ml}$ B27 (Sigma), $20 \mu \mathrm{g} / \mathrm{ml}$ gentamicin (Sigma), 1\% 100× Glutamax (Sigma), and 0.6\% glucose] at $37^{\circ} \mathrm{C}, 5 \% \mathrm{CO}_{2}$ and $95 \%$ humidity up to day in vitro (DIV)5. The medium was then switched to changing medium [MEM supplemented with $10 \%$ HS and $0.5 \%$ 5-fluorodeoxyuridine (FUDR)] to limit glial cell division. From DIV8 onwards, cultures were maintained in final medium (MEM supplemented with $10 \% \mathrm{HS}$ ) with a periodic fluid replacement every $3 \mathrm{~d}$. The neuronal cultures contained both excitatory and inhibitory connections, 
which were left active in all measurements to maximize spontaneous activity.

A total of $n=14$ cultures was used in this study. They were selected from all the pool of available cultures to comply two main conditions, namely a high spontaneous activity and a similar number of clusters. All experiments were conducted at $20^{\circ} \mathrm{C}$.

\section{Imaging setup}

Minicultures were imaged in pairs at DIV9-DIV13, a developmental stage in which the number and position of the clusters was stable and spontaneous activity high. Neuronal activity was monitored through fluorescence calcium imaging using Fluo-4-AM as $\mathrm{Ca}^{2+}$ probe (Teller et al., 2014, 2015). Prior recording, cultures were incubated for $25 \mathrm{~min}$ in a transparent, $\mathrm{pH}$-stable medium (recording solution, RS) that contained $2 \mu \mathrm{g}$ of Fluo-4 per milliliter of solution. At the end of incubation and after washing off residual Fluo-4, the cultures were transferred to an observation chamber that contained $2 \mathrm{ml}$ of RS. The chamber was sealed with a glass coverslip to prevent evaporation and left $5 \mathrm{~min}$ in darkness for stabilization.

The observation chamber was mounted on a multimodal microscope attached to a high-speed sCMOS camera (Hamamatsu Orca Flash 4, USB3 mode) that allowed for the simultaneous imaging of two minicultures. The multimodal microscope is a modified commercial confocal microscope (Nikon C1) that integrates a femtosecond-pulsed laser source for two-photon fluorescence microscopy (Mathew et al., 2009). This pulsed laser input was optimized for accurate multiphoton microsurgery and optical manipulation/stimulation of biological samples (Santos et al., 2013).

\section{Laser microsurgery}

Optical surgery in combination with fluorescence imaging was achieved by setting the microscope in three progressive configuration modes, termed top EPI, laser, and bottom transmission. Fluorescence imaging of spontaneous activity was recorded using the top EPI configuration. Here, the multimodal microscope operated as an epifluorescence microscope in an upright configuration. A mercury lamp (Nikon C-HGFI) guided by an optical fiber was coupled onto the EPI-illumination port. The green fluorescence protein (GFP) filter set for Fluo-4 $\mathrm{Ca}^{2+}$ imaging consisted of a dichroic mirror (FF 509 FDI) with a green filter and a blue bandpass filter (HQ470/40X). Frames were acquired with a size of $534 \times 254$ pixels $(6.5$ $\times 3.5 \mathrm{~mm}^{2}$ field of view), 16-bit grayscale, and acquisition speeds in the range of 83-100 frames/s (fps).

Neuronal clusters were targeted using the laser configuration. A Ti:sapphire laser (Mira Optima 900-F, Coherent) producing an ultrashort (150 fs) near-infrared (NIR) pulsed beam, with an average power of $400 \mathrm{~mW}$ in the back focal plane of the objective, was delivered onto a region of 0.7 $\mu \mathrm{m}^{2}$. In this setting, the laser light was focused using a NIR-optimized water immersion objective with 1.05 numerical aperture ( $25 \times$, Olympus). A shutter was incorporated between the attenuators to control the exposure time of the laser.
Transmission bright field images of the cultures were obtained through the bottom transmission configuration. To minimize the time and changes in the custom setup, the standard bottom illumination from the EPI configuration was used together with the TRITC epifluorescence cube. The emission filter of the GFP cube of the top configuration transmitted the excitation light of the TRITC cube and produced a normal transmission image of the sample.

\section{Experimental procedure}

Neuronal spontaneous activity was evaluated through calcium fluorescence imaging. To select the most appropriate cultures, activity was first recorded for $5 \mathrm{~min}$ in the four minicultures PDMS set. The pair of minicultures that contained a comparable number of clusters and exhibited similar activity was selected, and the entire glass adjusted to fit this pair in the field of view. One of the cultures was then designated as control and the other one as target, and spontaneous neuronal activity recorded in the pair for 30 min.

The multimodal microscope was next switched to the laser configuration for microsurgery on a random cluster. With assistance of a second camera (DCC 1545M, Thor Labs) and a joystick, the laser beam was positioned on the cluster and manually guided. Damage was applied along the surface and edges of the target cluster, effectually killing all its neurons and disconnecting the cluster from the rest of the network. The microscope objective had a non-negligible chromatic aberration that produced a shift between the image obtained with the camera and the ablation IR laser. This shift was compensated by moving the focal position along the $z$-axis to the plane where the damage was induced. The duration of the entire ablation operation was $\sim 10 \mathrm{~min}$.

Finally, the microscope was reconfigured for florescence imaging and activity in the control and target culture recorded again for additional $30 \mathrm{~min}$. A bright field image of the twin minicultures was taken at the end of the experiment to obtain a detailed characterization of the neuronal clusters.

All procedures were always conducted on the pair of minicultures, one acting as control and the other as target. This ensured that all experimental manipulations, such as handling of cultures or changes in the optical configuration, were experienced by both cultures. This was particularly important in the context of the laser ablation, in which the long time of the procedure as well as temperature variations associated to laser power could alter spontaneous activity. Post hoc data analysis showed that the control cultures exhibited stable characteristics along the experimental pipeline, and that therefore all network changes observed in the ablated culture originated from the physical damage and not from the experimental manipulations.

\section{Fluorescence signal and onset times}

Fluorescence recordings were first converted into individual frames using Hokawo 2.5 software (Hamamatsu). Neuronal clusters were manually selected as regions of interest (ROls) over the images to extract their fluorescence intensity (average grayscale level) along the re- 
corded frames. A typical experiment contained on the order of $40 \mathrm{RO}$ ls with a typical size of $40 \times 40$ pixels. The raw fluorescence signal of each neuron $F(t)$ was then corrected for small drifts by detrending the signal, i.e., by fitting a straight line to the baseline and subtracting it from the data. The detrended signal was then normalized as $\operatorname{DFF}(\%) \equiv 100 \times\left(F-F_{0}\right) / F_{0}$, with $F_{0}$ the fluorescence level of the neuron at rest. The normalized fluorescence signal was analyzed to determine the onset times of activation, characterized by a sharp increase of the fluorescence signal of the clusters. Following previously described algorithms (Teller et al., 2014, 2015), onset times were detected as the first occurrence of the crossing between the cluster's fluorescence signal and a threshold value set as two times the average fluorescence signal of the cluster.

\section{Firing sequences}

Spontaneous activity in clustered networks is characterized by the concatenated activation of two or more clusters in a short time window. These activations, termed firing sequences (Teller et al., 2015), provided the basis for the computation of the effective connectivity of the networks and its modular organization. Following previous studies (Teller et al., 2014, 2015), two or more clusters belonged to the same firing sequence when their coactivation time delay was $<200 \mathrm{~ms}$. For simplicity, we will also use the term "firings" to refer to these firing sequences.

\section{Network construction}

\section{Effective connectivity computation}

The effective connectivity was computed either along the entire recording or along a sliding time window. The degree of coupling among pairs of clusters within a firing sequence was asserted through time delays (Teller et al., 2014). In this approach, the more frequently two clusters coactivate together, the stronger their connection weight, and with the directionality of the interaction given by the temporal order of coactivation. This approach provided a connectivity matrix $\mathbf{A}=\left\{a_{i j}\right\}$ that was thus weighted and directed. A null model was used to evaluate the significance of the inferred effective links and to normalize the connectivity matrix. The null model consisted in a random permutation of the times of the firing events of each cluster's time series (Teller et al., 2014, 2015). This method erased the temporal correlations among firing clusters but preserved the average network activity. A total of 500 surrogates were generated, each one procuring a connectivity matrix $\mathbf{A}^{\mathbf{s}}=\left\{a_{i j}^{S}\right\}$. Significant links $\mathbf{Z}=$ $\left\{z_{i j}\right\}$ were then set according to the $z$-score

$$
z_{i j}=\frac{a_{i j}-\left\langle a_{i j}^{S}\right\rangle}{\sigma_{i j}^{S}},
$$

where $\left\langle a_{i j}^{S}\right\rangle$ is the average surrogates' weight between clusters $i$ and $j$, and $\sigma_{i j}^{S}$ the corresponding standard deviation (SD). High values of $z_{i j}$ reflected strong cluster-tocluster interactions. Negative $z_{i j}$ values indicated links that were less connected than in a random configuration, which were disregarded and set to 0 . The $z$-score implementation of Equation 1 quantified the difference, in SD units, between the cluster's raw connectivity value and the surrogates' av- erage value. The $z$-score defined a fixed reference to compare different cultures and experimental conditions and did not require the selection of an arbitrary threshold for significance.

The final set of effective links' weights $\mathbf{W}=\left\{w_{i j}\right\}$, from which all network measures were computed, was set as

$$
w_{i j}=\frac{z_{i j}}{\max \left(z_{i j}\right)},
$$

thus procuring a normalized effective connectivity matrix with values in the range $[0,1]$. This normalization facilitated the comparison and averaging among experiments. We verified that the procured effective connectivity matrix $\mathbf{W}$ using our time-delays approach was similar, in number of effective links and computed network measures, to the one obtained using other approaches such as transfer entropy (Stetter et al., 2012).

\section{Network dynamic evolution}

Effective connectivity matrices at different time points were constructed to monitor the time-varying behavior of the clustered cultures before and after damage. A sliding window approach (Sakoğlu et al., 2010; Kiviniemi et al., 2011; Jones et al., 2012; Allen et al., 2014) was used to compute the effective connectivity matrices. Time window of length $\Delta t$ (centered at time $\tau$ ) progressively scanned the recording without overlap. The set of firing sequences within each window was then analyzed to infer the effective connectivity matrices $\mathbf{W}^{\tau}$. The mean firing rate of the cultures before and after damage was typically four and three firings per minute, respectively. Since a minimum number of five firing sequences was required for a reliable inference of $\mathbf{W}^{\tau}$, the window size was set in the range $2.5<\Delta t<4 \mathrm{~min}$ in both cases. An inspection of all the experiments showed that this setting provided $\sim 5-12$ firing sequences per window. The number of windows was therefore given by $T / \Delta t$, where $T=30 \mathrm{~min}$ is the duration of the recording, leading to 9-12 windows for the analysis of the "before" and "after" damage conditions.

\section{Network measures}

They were computed on the time-windowed effective connectivity matrices using the Brain Connectivity Toolbox (MATLAB; Rubinov and Sporns, 2010). From here on, $N$ indicates the total number of nodes in the network. The ablated node was always excluded in the analysis, both before and after damage, to prevent a bias associated to network size.

\section{Nodal strength and total network strength}

The nodal strength $s_{i}$ was defined as the sum of all input and output weights to node $i, s_{i}=\sum w_{i j}$. The average nodal strength $\bar{s}$ was the mean of all nodal strengths, $\bar{s}=(1 / N) \sum s_{i}$. The total network strength $S^{\text {net }}$ accounted for the sum of all nodal strengths or, equivalently, the sum of all weights, $S^{\text {net }}=\sum_{i} s_{i}=\sum_{i j} w_{i j}$.

\section{Density of links D}

It was defined as the fraction of total existing weighted links to all possible $N(N-1)$ connections in the directed network. For a network with a total strength $S^{\text {net }}$, the 
density of links was then $D=S^{\text {net }} /(N(N-1))=\sum_{i j} w_{i j} /$ $(N(N-1))$.

Global efficiency $\mathrm{G}$

The efficiency $E$ of a network of $N$ nodes was calculated as (Rubinov and Sporns, 2010)

$$
E=\frac{1}{N(N-1)} \sum_{N=1}^{\infty} \frac{1}{d(i, j)},
$$

where $d(i, j)$ denotes the minimum topological distance connecting nodes $i$ and $j$. The global efficiency $G$ is the relative value $G=E / E_{c}$, where $E_{c}$ refers to the efficiency of a clique formed by the same number of nodes. $G$ provided a quantification of the communication among neuronal clusters and the integration capacity of the network.

\section{Time evolution of $\mathrm{D}$ and $\mathrm{G}$}

Control and ablated cultures were measured simultaneously in each recording, and analyzed identically. The time evolution of either $D$ or $G$ along the recording was introduced to quantify the impact of laser ablation and the recovery of the culture. Thus, $D$ and $G$ were analyzed along different time windows centered at $\tau$, as described above. Each condition (before damage or after damage) procured $\sim 10-15$ data points. Since the window centers $\tau$ varied across experiments, the curves $D(\tau)$ and $G(\tau)$ were interpolated in $1 \mathrm{~min}$ time steps. Data were then averaged among the $n=14$ experiments to provide the final $D(t)$ and $G(t)$ curves, with $t=\{1,2 \ldots, T\}$ min.

\section{Integrability loss $\Lambda$}

It provided the relative loss in global efficiency for the ablated culture following damage. It was computed for each culture as

$$
\Lambda(\%)=100 \times \frac{\tilde{G}^{\text {bef }}-G_{0}^{\text {aft }}}{\tilde{G}^{\text {bef }}},
$$

where $\tilde{G}^{\text {bef }}$ is the time-averaged global efficiency of the culture before damage (with SD SD bef), and $G_{0}^{\text {aft }}$ is the first measured value of the global efficiency just after damage.

Integrability recovery rate $\Theta$ and recovery time $T_{R}$

$\Theta$ characterizes the typical increase of the relative global efficiency along time during recovery. It was computed for each ablated culture as $\Theta(\%)=\Lambda(\%) / T_{R}$, where $T_{R}$ is the time required for the culture to attain the global efficiency before damage. $T_{R}$ was determined as the moment in which the global efficiency along recovery $G$ aft $(t)$ first reached $\tilde{G}^{\text {bef }}-S_{G}^{\text {bef }}$.

\section{Neighborhoods of clusters around damage}

Six neighborhoods of progressively distant rings from damage were defined. The clusters belonging to the first neighborhood were those located at a distance below a radius $r_{C} \simeq 0.68 \mathrm{~mm}$ from the ablated cluster. This radius was set as the average intercluster separation and was the same for all cultures. The second and further neighborhoods were formed by those clusters located at a distance $r_{C}$ from the previous ring and away from damage.

\section{Interaction probability $\mathrm{P}$}

It accounted for the probability to observe intraneighborhood and interneighborhood effective links. Conceptually, $P$ rendered the capacity of a pair of neighborhood rings $R_{u}$ and $R_{v}$ to project effective connections to one another. $P$ was computed as

$$
P\left(R_{u}, R_{v}\right)=\sum_{i, j \in R_{u} \cup R_{v}} w_{i j} / M,
$$

where $i$ and $j$ are the indexes of the clusters encompassing rings $R_{u}$ and $R_{v}, w_{i j}$ their weight, and $M$ all the possible directed links that can be formed between and within $R_{u}$ and $R_{v}$.

Flow of links $\mathrm{F}$ and percentage variation of flow of links $\mathrm{F}^{*}$

The flow of links $F$ quantified the fraction of weighted links that flowed between two neighborhood rings $R_{u}$ and $R_{v}$. $F$ was computed in two steps. In a first one, the percentage $C\left(R_{u}, R_{v}\right)$ of links between rings $R_{u}$ and $R_{v}$ with respect to all links in which ring $R_{u}$ participates was calculated as

$$
\begin{gathered}
C\left(R_{u}, R_{v}\right)=\frac{\text { links between rings } R_{u}, R_{v}}{\text { all links connecting } R_{u} \text { with any other ring }} \\
=\frac{\sum_{(i, j) \in\left(R_{u} \cup R_{v}\right)} w_{i j}}{\sum_{v \neq u} \sum_{(i, j) \in\left(R_{u} \cup R_{v}\right)} w_{i j}} .
\end{gathered}
$$

In a second step, $F$ was determined as $F\left(R_{u}, R_{v}\right)=C$ $\left(R_{u}, R_{v}\right) / P\left(R_{u}, R_{v}\right)$, where $P$ is the interaction probability. This operation established $F$ as a normalized measure that facilitated the averaging among different networks. The values procured by $F$ for each ring pair were used to show the behavior of the network before damage, and were denoted $F_{\text {bef }}$. The behavior of the network just after damage and in subsequent temporal windows $\tau$ along recovery was portrayed through the percentage variation of flow of links $F^{*}$, given by

$$
F^{*}(\tau)=100 \times \frac{F(\tau)-F_{\text {bef }}}{F(\tau)+F_{\text {bef }}},
$$

where $F(\tau)$ and $F_{\text {bef }}$ are, respectively, the flow of links at a given temporal window $\tau$ and before damage. We considered only the values of $F^{*}>0$ to emphasize the flow of new effective links. This helped highlighting those rings that increased the number of effective links with respect to the predamage condition.

\section{Statistical analysis}

Statistical and graphical analyses were conducted with Origin 9.1 and Prism 8 software packages. One-way ANOVA was used to analyze the following: (1) the differences between global efficiency and density of links before and after damage and (2) the difference in activity levels between neuronal neighborhoods along recovery. Statistical significance was designated at $p=0.05$ for all analyses. When appropriate, data were represented and examined via box plots. 

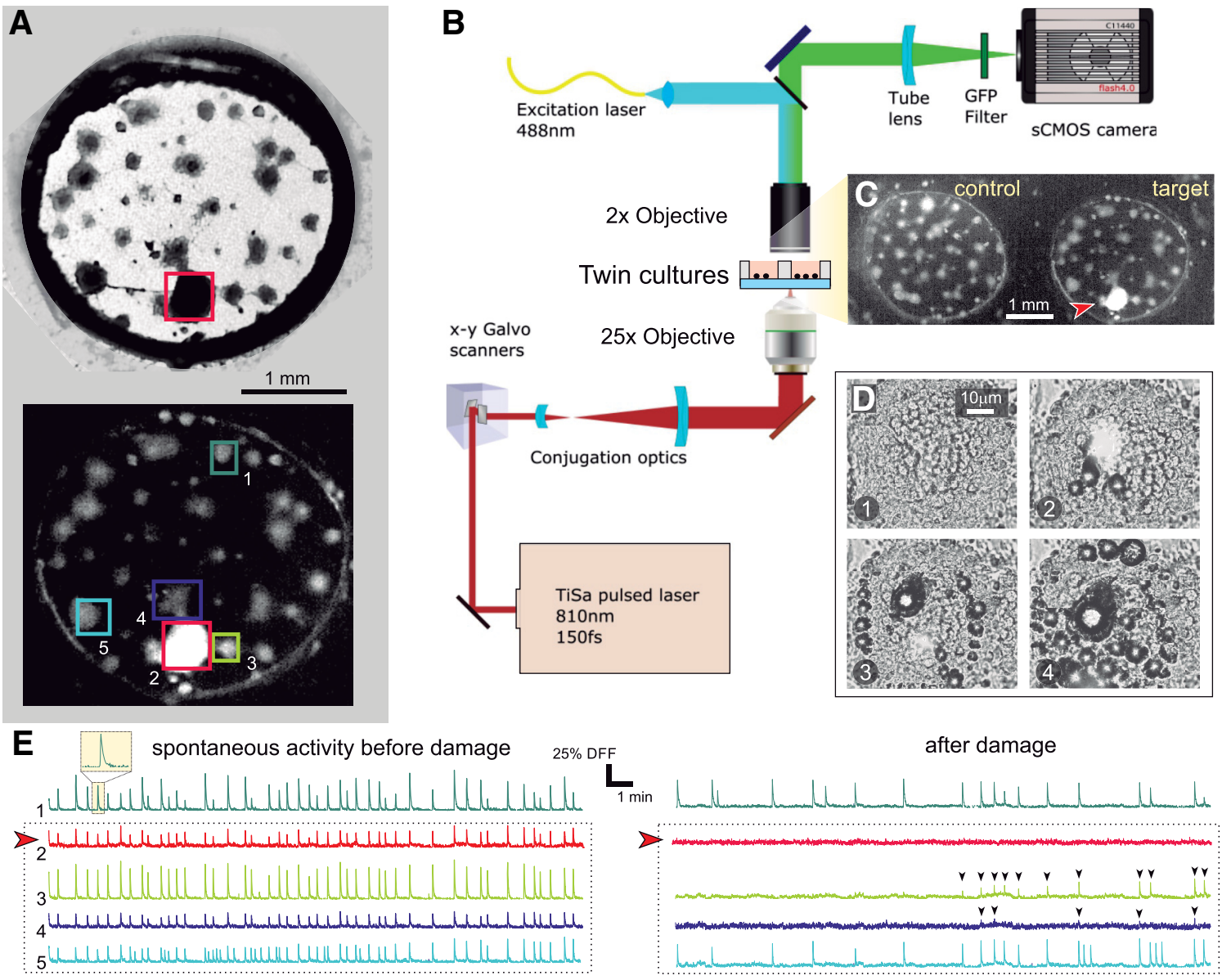

Figure 1. Clustered neuronal cultures and experimental procedure. $\boldsymbol{A}$, top, Bright field image of a clustered neuronal culture $3 \mathrm{~mm}$ in diameter. Dark circular objects are neuronal clusters, and straight filaments are connections. The ablated cluster is boxed in red. Bottom, Corresponding fluorescence image after damage. Healthy clusters appear gray. The ablated cluster, with all its neurons dead, appears bright. Boxed clusters are those whose spontaneous activity is represented in panels $\boldsymbol{E}, \boldsymbol{F}$. B, Sketch of the multimodal optical system for fluorescence imaging and laser microsurgery. $\boldsymbol{C}$, Actual field of view in the experiments. Two cultures are simultaneously monitored, with one set as control and the other one as target. The latter is the same culture as in panel $\boldsymbol{A}$, and the red arrowhead signals the ablated cluster. $\boldsymbol{D}$, Laser microsurgery. The four snapshots illustrate the action of the laser as it progressively scans the cluster to be ablated, delivering in each step a high energy, high penetration pulse that kills the neurons and vaporizes water. The time interval between panels is $20 \mathrm{~s}$. $\boldsymbol{E}$, Spontaneous activity before damage for the five clusters highlighted in $\boldsymbol{A}$. Activity is rich and all clusters fire together in a highly coordinated manner. The red arrowhead marks the cluster to be ablated. $\boldsymbol{F}$, Corresponding activity after damage, with the ablated cluster completely silent. Its immediate neighbors are initially silent but recover activity after $\sim 10 \mathrm{~min}$, although with lower firing rates and amplitudes (black arrowheads). Clusters more distant from damage maintain their activity after ablation, although with a reduced firing rate.

\section{Results}

\section{Focal damage on neuronal cultures}

We investigated the response of neuronal cultures to the destruction of a node in the network through laser ablation. Cultures were an ensemble of interconnected neuronal aggregates termed "clusters" grown on 3-mm diameter PDMS cavities. As shown in Figure $1 A$, a typical culture contained $\sim 40$ quasi-spherical clusters with diameters in the range $50-200 \mu \mathrm{m}$, which connected to one another through bundles of axons that appeared as straight filaments. We monitored spontaneous activity in these cultures using fluorescence calcium imaging, a technique that revealed neuronal activations as a sharp increase in the fluorescence signal followed by a slow decay to basal levels (Fig. 1A,E,F).
To monitor the response of a culture to damage we used a multimodal microscope that integrated two operational modes, a first one dedicated to calcium imaging and a second one dedicated to precision laser surgery. Figure $1 B$ outlines the microscope operational modes and the experimental procedure. The key advantage of the multimodal microscope is that activity monitoring and physical damage were integrated in the same system, minimizing time delays between operations and ensuring that changes in neuronal network behavior were solely ascribed to physical damage and not to other manipulations.

In a typical experiment, spontaneous activity was first monitored for $30 \mathrm{~min}$ in a pair of cultures (Fig. 1C), which were previously selected according to their similarity in 
A

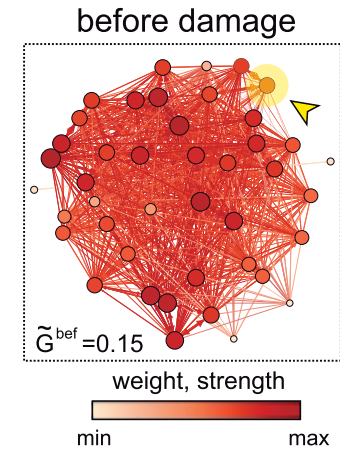

B
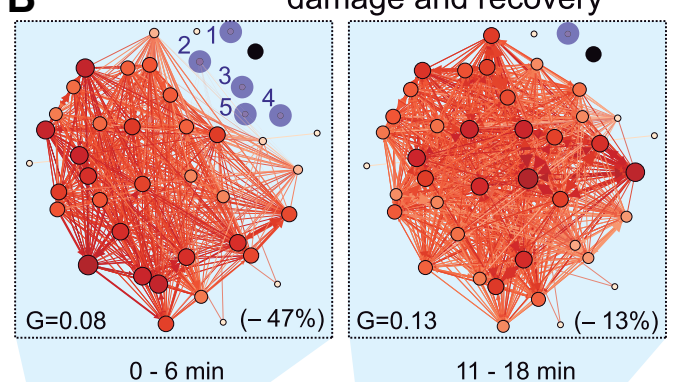

$11-18 \mathrm{~min}$

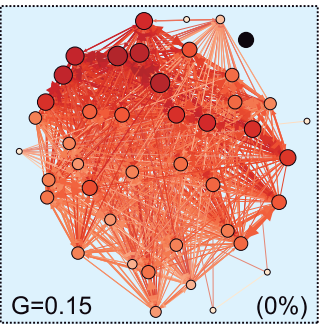

$23-28 \mathrm{~min}$
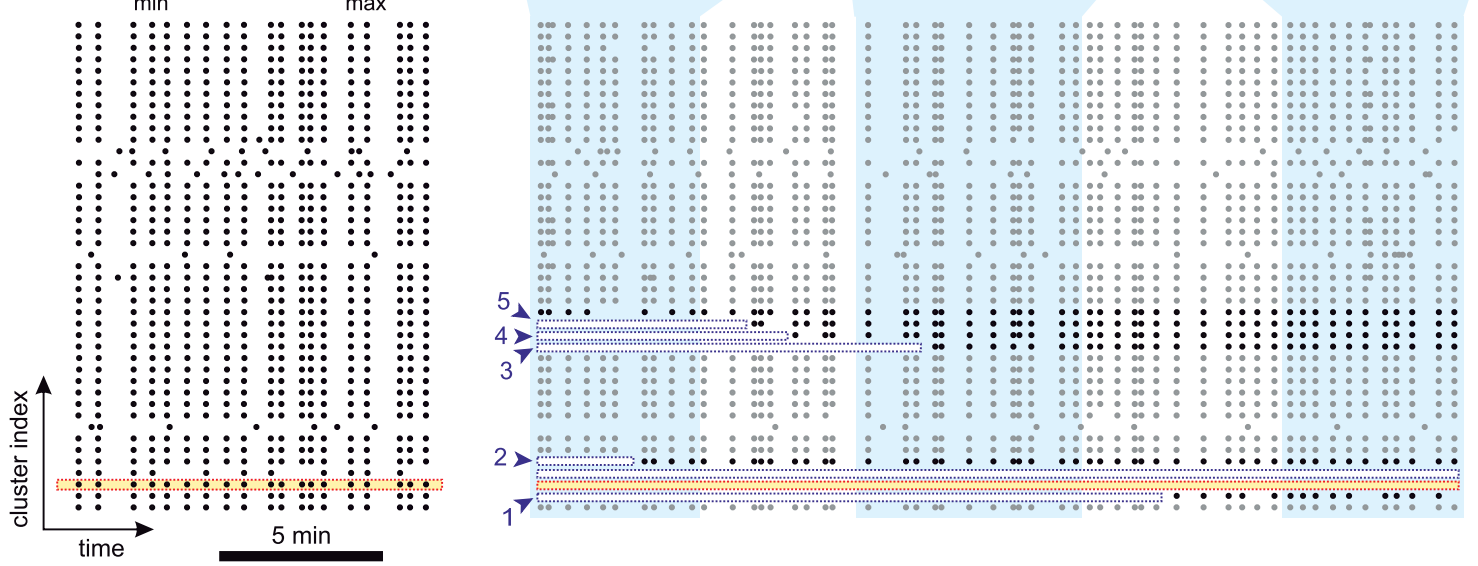

Figure 2. Network evolution during recovery. $\boldsymbol{A}$, Network effective connectivity and raster plot of activity before damage. The effective connectivity is computed on the full, $30 \mathrm{~min}$ duration of the recording. Nodes and links are color coded according to their strength and weight, respectively. The darker the color, the higher the value. The yellow arrowhead marks the targeted cluster. G bef provides the global efficiency before damage. The bottom raster plot shows the 10 min before damage, with the yellow band highlighting the cluster to be ablated. Black dots are activations. B, Effective connectivity evolution and raster plot after damage. The effective connectivity networks were computed in $\sim 6$ min time windows. The ablated cluster is marked in black. Clusters in blue are those that became silent just after damage but recovered afterward, with the numbers indicating their location in the bottom raster plot. $G$ provides the global efficiency, and its relative change with respect to $\tilde{G}$ bef is shown in brackets. In the raster plot, the ablated cluster is shown with a yellow band; the initially silent clusters are shown with a white band. One of these clusters never recovered and the band encompasses the full duration of the raster plot. Gray dots are activations in clusters that did not substantially change activity after damage. Black dots are activations in affected clusters.

number of clusters and activity. Next, one of the cultures was left unaltered as control, while the other was damaged by ablating a preset cluster from the rest of the network (Fig. 1C, arrowhead). Ablation was achieved through a high-power pulsed laser that scanned the entire volume of the cluster with micrometer resolution, locally increasing the temperature and generating vapor bubbles (Fig. 1D). Both effects led to neuronal death inside the target cluster. At the end of the process the ablated cluster appeared markedly bright and had no activity, signatures of full damage (Fig. $1 A, C$ ). The subsequent evolution of the pair of cultures was then monitored for an additional $30 \mathrm{~min}$.

The changes in spontaneous activity before and after damage are shown in Figure. $1 E, F$, which depict the fluorescence traces for the boxed clusters of Figure $1 A$. Before damage (Fig. 1E), all clusters exhibited a strong coordinated activity, firing periodically together in the same time window. These episodes of high intercluster coordination reflected the strong coupling of the network. After damage (Fig. 1F), the trace of the ablated cluster contained just noise. Remarkably, the nearest neighbors to the ablated cluster were silent for the first $10 \mathrm{~min}$ to gradually restore activity afterward. The abrupt silencing of the clusters at the vicinity of the ablated one evinces the strong impact of focal damage on the immediate neighborhood, a feature that was observed in all experimental realizations. Although distant clusters decreased activity in the experiment shown here, such a long-distance affectation was rare.

\section{Evolution of effective connectivity after damage}

To evaluate the alterations caused by damage, we considered a representative culture and computed the effective connectivity of the network along different time windows. According to the choice for effective connectivity inference, the more frequently two clusters coactivate together, the stronger the connection weight. Thus, before damage (Fig. $2 A$ ), the high level of coordination among firing clusters procured a strongly coupled effective network, in which the clusters with the highest strength (total incoming and outgoing weighted effective connections) were uniformly spread. 
The ablation of the target cluster (Fig. 2A, arrowhead) precipitated different events at both spatial and temporal scales. Firstly, as shown in the raster plot of Figure $2 B$, not only the targeted cluster became silent (yellow band), but also its immediate neighbors (blue arrows, white bands). Secondly, some of these affected clusters recovered activity to levels previous to damage in $\sim 10-15 \mathrm{~min}$, a feature that suggests the activation of fast recovery mechanisms. And thirdly, the effective networks markedly changed in organization following damage. Indeed, just after ablation the links with the highest strength appeared far from the damaged region to progressively concentrate around it. Since the strength of a node reflects its degree of interaction with neighbors, the recovery of the network is associated to an increase in intercluster activity around damage.

The behavior of the network as a whole was quantified through the global efficiency $G$ (Rubinov and Sporns, 2010), which measures the degree of integrability in the network, i.e., its capacity for broad communication and information exchange. Before damage (Fig. 2A), the average global efficiency of the network was $\tilde{G}^{\text {bef }} \simeq 0.15$, which dropped to $G \simeq 0.08$ just after damage (Fig. $2 B$ ). These values provided an integrability loss of $\simeq 47 \%$. The global efficiency gradually increased afterward and the network (excluding the ablated cluster) attained full recovery $\sim 15$ min after damage.

\section{Evolution of global efficiency and density of links}

To prove that functional recovery was a general feature, we investigated a total of 14 cultures of identical size, containing an average number of $40 \pm 5$ clusters and a similar spontaneous activity of $4 \pm 1$ firings $/ \mathrm{min}$. In all cases only one node was ablated. We used the global efficiency $G$ and the density of effective links $D$, averaged over experimental realizations, as main descriptors for network behavior. The global efficiency reflects whole network integrability, as seen before. The density of links, defined as the fraction of existing weighted links with respect to all possible links, portrays the degree of dynamic interactions among clusters. Figure $3 A$ shows the average evolution of the global efficiency and density of links for the 14 ablated cultures and their controls. For clarity, the presented data corresponds to the $10 \mathrm{~min}$ before damage and to the $15 \mathrm{~min}$ just after damage.

The global efficiency $G$ (Fig. $3 A$, top panel) exhibited a stable behavior within experimental variability, and both control and ablated cultures procured similar values of $G$. After damage, controls maintained an overall stable behavior, although fluctuations were higher probably due to the changes in the optical setup along the laser ablation procedure, which slightly increased the temperature of the recording chamber. Ablated cultures, however, experienced a substantial drop in $G$ at damage, by $50 \%$, but gradually recovered afterward and attained values of $G$ very similar to predamage levels in $\sim 10 \mathrm{~min}$. Concurrently, the density of effective links $D$ (Fig. $3 A$, bottom panel) also showed a stable behavior for control and ablated cultures before damage, procuring similar values of $D$ despite fluctuations. $D$ dropped on damage due to the loss of activity around the ablated cluster, to gradually increase afterward as activity returned.

Both $G$ and $D$ exhibited strong variability on recovery (Fig. 3A, high SDs), with $D$ even exceeding the average value before damage. We ascribe the high variability to the characteristic connectivity blueprint of each culture, which led to different interactions among clusters and therefore broad values of $G$ or $D$ at a given time point. On the other hand, we view the high average value of $D$ as a signature of the recovery process itself, in which plasticity mechanisms that compensate for the loss of activity are activated, increasing the number and strength of effective links among clusters.

The density of links $D$ exhibited interesting traits at a network level. The circular panels accompanying Figure $3 A$ depict effective networks at three representative time windows of the experiment shown in Figure 2. The networks spotlight the location of the $5 \%$ strongest effective links, thus portraying the most frequent cluster's coactivations. Before damage, the strongest links were well spread across the network and involved nearby clusters. Immediately after damage, the strongest links appeared far from the ablation core and involved distant clusters. As recovery took action, the strongest links predominated around the ablated cluster and as shortrange interactions. These changing dynamic scenarios illustrate the complexity of the recovery process and that encompasses the creation of new connectivity pathways or the strengthening of existing ones.

To show in more detail the changes in $G$ and $D$ in the 14 cultures before and after damage, Figure $3 B$ compares in the form of box plots the behavior of the cultures at different stages. Data includes the controls before and after damage as well as the ablated cultures before damage, the window of 5 min just after damage ("aft,") and the window of 15 min before the end of recording and that encompasses full recovery ("aft ${ }_{R}$ "). A statistical comparison of the box plots indicated that the distributions of $G$ and $D$ values on ablation are significantly lower $(p<$ 0.001 , one-way ANOVA) than any other experimental condition. Thus, the alterations that the cultures experienced on ablation and subsequent recovery are associated to intrinsic network changes, and not to experimental details such as the number of clusters, their spatial distribution, or the culture age in vitro.

As an additional analysis, we quantified the degree of damage and recovery for each individual culture, and introduced three measures, namely the integrability loss $\Lambda$, the recovery time $R_{T}$ and the recovery rate $\Theta$. These measures were extracted from the evolution of the global efficiency $G(t)$ for each ablated culture, as illustrated in Figure $3 C$ for one particular culture. The distributions of these measures for the 14 explored cultures (Fig. 3D) displayed a broad range of values. On average, the measures indicated that global efficiency decayed by $\sim 80 \%$ on damage, and that recovery was attained in $\sim 12 \mathrm{~min}$ at a rate of $6 \%$ increase in global efficiency per minute. We also observed that there was no correlation between any of these measures and experiment-specific characteris- 

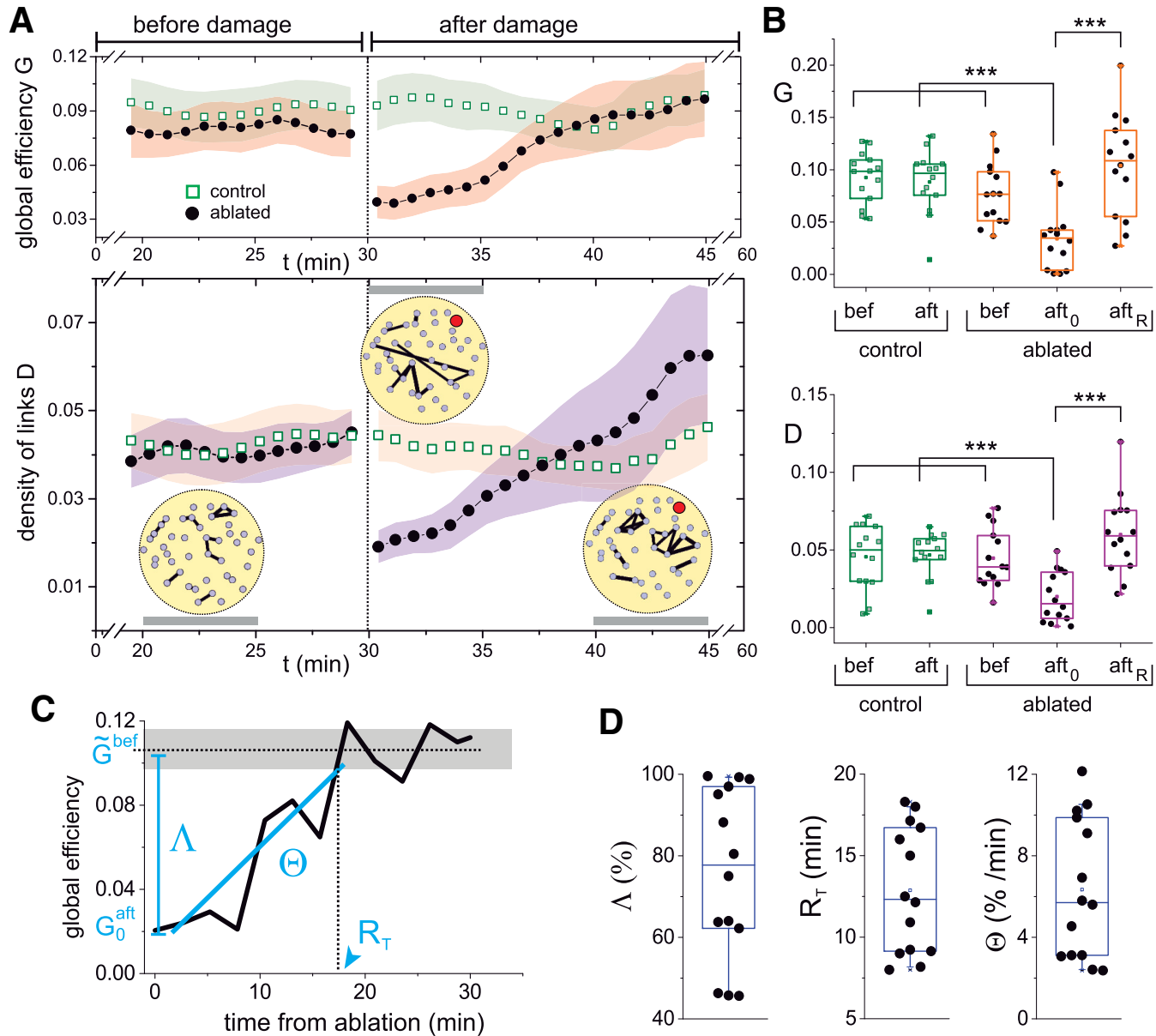

Figure 3. Variation of the global efficiency and the density of links on damage. $\boldsymbol{A}$, top plot, Time evolution of the global efficiency $G$ for control (green) and ablated (black) cultures, before and after damage. Bottom plot, Corresponding density of links $D$. The yellow panels provide representative effective networks of the experiment shown in Figure 2, are computed over $\sim 5$-min time windows (gray horizontal bars) and are thresholded to show the $5 \%$ links with the highest weight. The ablated cluster is marked in red. The networks illustrate the important changes in the distribution of links' weights along the recovery process. In both plots, data were averaged over 14 cultures and the shadings show SD. For clarity, only the last 10 min before damage and the first 15 min after damage are shown. $\boldsymbol{B}$, Box plots of the distribution of $G$ (top) and $D$ (bottom) values for the 14 cultures at different experimental conditions, comparing controls before and after damage with ablated cultures before damage, the first 5 min after ablation (aft t $_{0}$ and the last 15 min of the recording and that correspond to the recovered state $\left(\mathrm{aft}_{R}\right)$. For both $G$ and $D$, significance (*** $p<0.001$, one-way ANOVA) is only observed between the condition just after damage and the rest of conditions. $\boldsymbol{C}$, Evolution of the global efficiency for a representative individual experiment to spotlight the definitions of the global efficiency loss $\Lambda$, recovery time $R_{T}$, and recovery rate $\Theta$. $\tilde{G}^{\text {bef }}$ and $G_{0}^{\text {aft }}$ are, respectively, the global efficiencies before damage (dotted line for average, gray shading for SD) and just after damage. $\boldsymbol{D}$, Distributions of $\Lambda, R_{T}$, and $\Theta$ for all 14 experimental realizations. All box plots span from the median to the first and third quartiles, and whiskers span from the 10th to 90th percentile.

tics such as the DIV of the culture or the location of the ablated clusters.

\section{Activity in the neighborhood of damage}

The results shown in Figure $3 A$ evinced the pivotal role of clusters' activity during recovery, which translated into the emergence of strong effective links at the vicinity of damage. To better understand this role, we investigated clusters' activity in progressively distant neighborhoods with respect to the damaged region. As illustrated in Figure $4 A$, the clusters that shaped the ring of "first neighbors" were those centered at a distance below a characteristic radius $r_{C} \simeq 0.68 \mathrm{~mm}$ from the ablated cluster. The second and further neighborhood rings were formed by those clusters located at a distance $r_{C}$ from the previous ring and away from damage.

For each neighborhood we plotted the average clusters' activity at different time steps, and encompassing all 14 experimental realizations. As shown in Figure $4 B$, we inspected activity before damage, just after damage, and along different recovery stages. The analysis of the data procured two major results. On the one hand, damage had a strong impact on activity in all neighborhoods, as indicated by the significantly different distributions of clusters' activity values before and after damage $(p<$ 0.05 , one-way ANOVA). Activity dropped by $74 \%, 49 \%$, and $41 \%$ for the first, second, and third neighborhoods, respectively. The remaining neighborhoods reduced ac- 
A

1st neighborhood

( $12 \%$ of clusters in the culture)

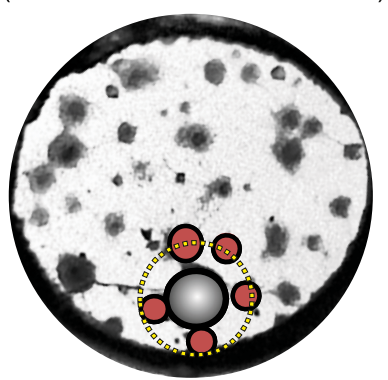

B
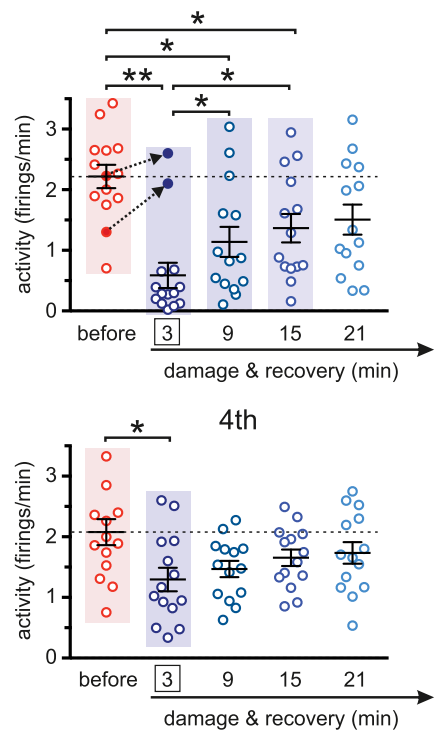

2nd neighborhood (19\%)

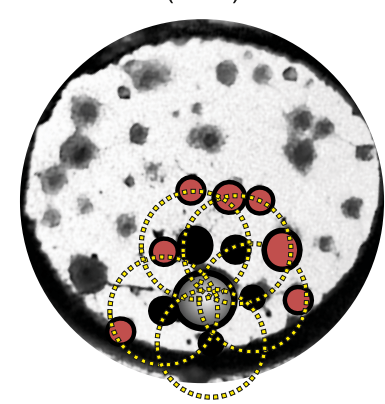

2nd neighborhood
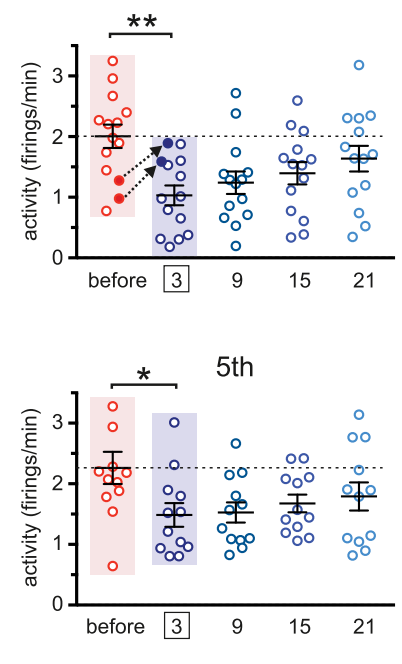

3rd neighborhood $(20 \%)$

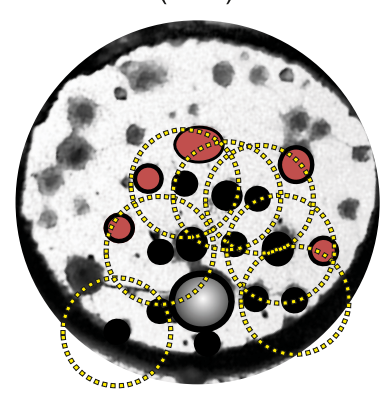

3rd neighborhood
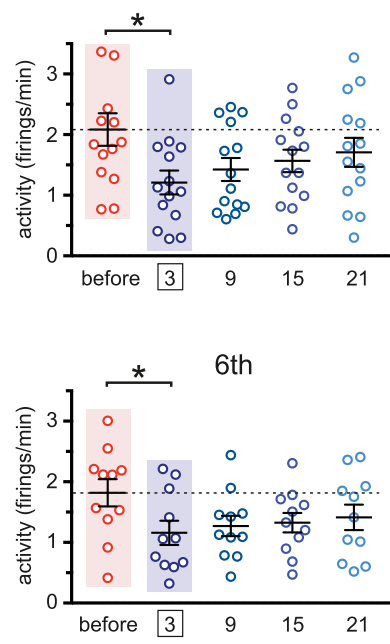

Figure 4. Spontaneous activity in the neighborhood of damage. $\boldsymbol{A}$, Construction of the ring of neighbors for a representative culture. The first neighborhood ring (red clusters) is constituted by all clusters whose centers fall within a distance $R_{C}=0.68 \mathrm{~mm}$ (yellow circle) from the ablated cluster (gray). The second and subsequent rings are built by identifying the clusters that are neighbors of the clusters in the previous ring according to the same distance $R_{C}$. $B$, Box plots showing the temporal evolution of the average activity in six neighborhoods and for the 14 experimental realizations. Before damage, activity is averaged over 30 min. The indicated times correspond to the center of the analysis windows $t$. For damage (at $t=3 \mathrm{~min}$, boxed) and subsequent recovery stages, activity is averaged in 6-min windows. Dotted black arrows highlight two experiments whose activity boosted up after damage. Average values of the distributions are shown as mean \pm SD. The colored panels highlight the distributions that are significantly different according to a one-way ANOVA ( $* p<0.05, * * p<0.01)$.

tivity by $30 \%$. On the other, once recovery took action, activity in the neighborhoods gradually increased along time, although at different rates. The first and second neighborhoods, for instance, boosted activity by $100 \%$ (from 0.6 to 1.2 firings $/ \mathrm{min}$ ) and $30 \%$ (from 1.0 to 1.3 firings/min), respectively, 9 min after damage. The other neighborhoods also increased activity on average, but by a milder $10-20 \%$.

Activity data also showed experiment-specific traits that are worth pinpointing. Specifically, we identified two experiments in which activity in the first and second neighborhoods increased after damage (Fig. 4B, black arrows), and that suggests a sudden rerouting of activity flow across the network. This result illustrates the complexity of physical damage in neuronal circuits and the intricate structure-function relationship, in which local direct loss of neurons or synaptic connections does not necessarily trigger a cascade of failure at the vicinity of damage.

\section{Interaction among neighborhoods during recovery}

To complete the picture and gain further insight on the recovery process, we studied the degree of interaction within and between neighborhoods. This interaction was quantified through the probability $P$ of observing new effective connections among clusters that belong to the same or different neighborhoods. Data were computed for each culture and then averaged over cultures. As shown in Figure 5A, clusters' interactions before damage were strong and localized. Each ring of clusters exhibited a dense internal effective connectivity and was strongly coupled with its immediate neighbors. Damage caused an overall fall of clusters' interactions that affected most prominently the first and second rings around the ablated 
A

'Interaction probability' $P$ before damage
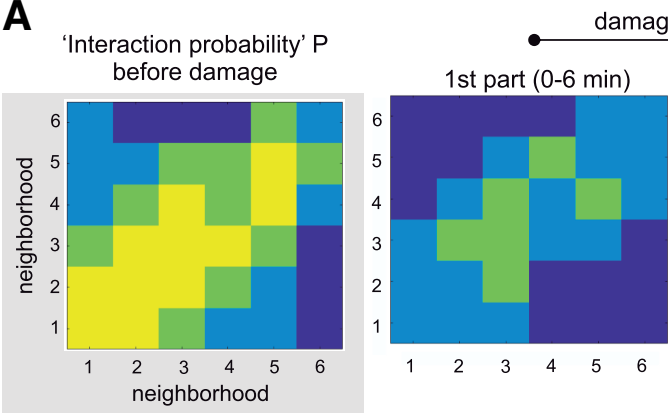

damage and recovery
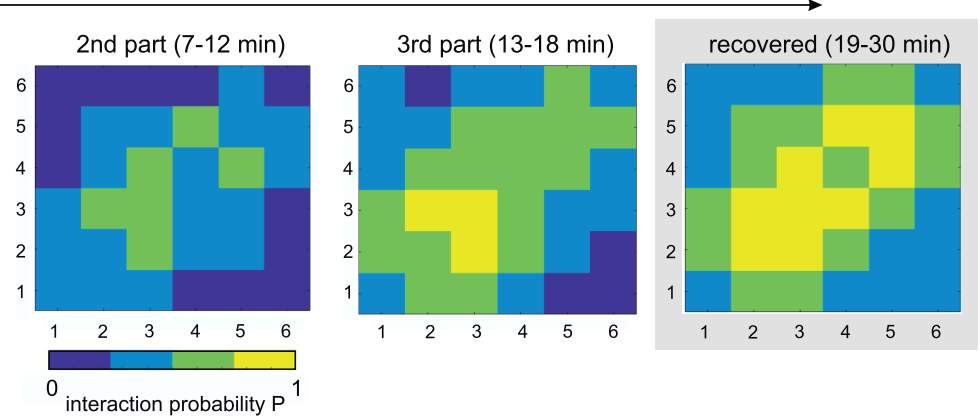

B
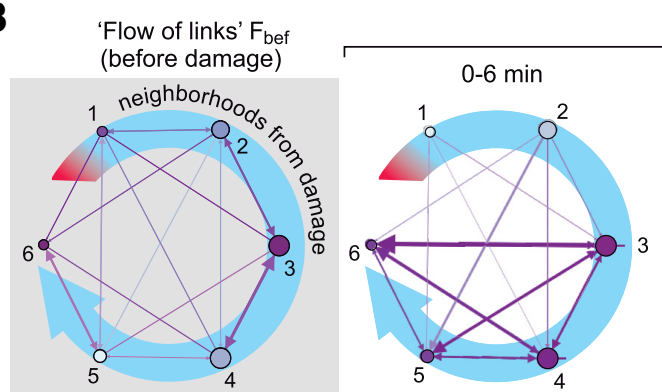

'Percent variation of flow of links' $F^{*}$ (with respect to pre-damage)

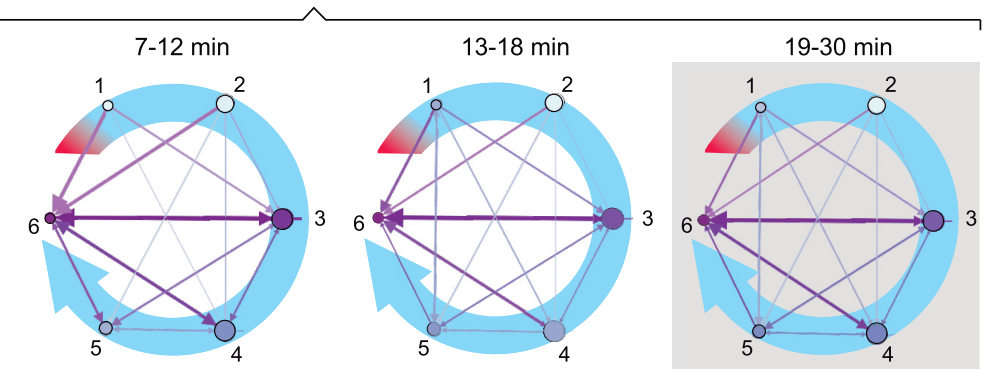

Figure 5. Network communication during recovery. $\boldsymbol{A}$, Interaction probability $P$ among all pairs of neighborhoods. The brighter the color, the higher the formation of effective links between and within neighborhoods. Data were computed for each culture and then averaged over the 14 studied cultures. The left panel shows the neighbors' interaction before damage, with data averaged over 30 $\min$. The three central panels show the action of damage and subsequent recovery, with each panel corresponding to $\sim 6$-min window intervals for analysis. The last panel shows the stationary recovery, with data averaged over a broader window of $\sim 12$ min. $\boldsymbol{B}$, Corresponding representation of the flow of links $F_{\text {bef }}$ (before damage) and the percentage variation of flow of links $F^{*}$ (rest of panels, calculated with respect to the predamage scenario). The blue curved arrow and the numbers indicate the distance from damage in terms of neighborhoods. Damage locus is symbolized as a red band. Before damage, purple arrows depict the communication flow between neighborhoods. After damage, the arrows depict the level of formation of new effective links between neighborhoods with respect to predamage. Arrows' thickness and color intensity are coded according to the values of $F$ or $F^{*}$. Nodes' color is coded according to the relative strength (weighted sum of incoming and outgoing links) of the neighborhood.

cluster. The third and fourth rings maintained a high degree of internal and external interactions, which gradually extended toward the rest of the network as recovery took place. These results suggest that the intermediate regions of the network, i.e., those that are neither too close nor too far from damage, initiated the recovery process. Remarkably, once recovery was attained, the interaction within and between neighborhoods was very similar to the one before damage. Only the first ring deviated from this trend. We argue that this ring was substantially sensitive to the physical wiring and activity drive of the ablated cluster, therefore substantially hampering ring's recovery.

Given the importance of interneighborhood communication during recovery, we analyzed in more detail the flow of effective links between neighborhoods. Figure $5 B$ portrays the directionality and degree of formation of effective links among neighborhoods. Before damage, the flow of links $F_{\text {bef }}$ shows that all six rings interacted among themselves with a similar degree, a result that is in agreement with the high coordinated activity of the clusters in the network. The action of damage, emphasized here by plotting the variation of flow of links $F^{*}$ with respect to the predamage condition, broke the uniformity of communication between neighborhoods and made ring 3 the leader in the formation of new effective links. Indeed, as the panels of Figure $5 B$ show, the beginning of recovery was characterized by a substantial flow of new links that either diverged from ring 3 or converged toward it, and essentially involving rings 4-6. This behavior reinforces the message that areas neither too close nor too far from damage (as ring 3 in our case) play a substantial role in maintaining network activity and leading recovery. In our experiments, as recovery progressed, rings 1 and 2 gradually participated more actively, shaping new effective links toward the rest of the network and balancing the entire system again. At full recovery, the interaction between neighborhoods procured an almost uniform formation of new links.

\section{Discussion}

The in vitro model presented here takes advantage of the accessibility and ease of manipulation of clustered neuronal cultures to investigate network's recovery and functional reorganization after acute focal damage. Our results show that the neuronal clusters adjacent to the lesion core, first and second neighborhood rings, were the most affected by damage, possibly due to the loss of direct physical connections. However, damage did not precipitate a cascade of failure. Clusters weakly linked to the lesion core, third and fourth rings, coped with damage 
and led recovery by establishing new functional connections or strengthening existing ones. Dynamic interactions extended next to the whole network to restore its communication to predamage levels.

In the experiments, the ablated cluster was chosen arbitrarily, and with the only condition that its level of activity was similar to the average of the network. For the 14 cultures studied, in $60 \%$ of the experiments the ablated cluster was located at the edge of the culture, and for the remaining $40 \%$ it was located at the center. We could not pinpoint any significant correlation between the location of the ablated cluster and the characteristics of recovery, indicating that the distance of the clusters to the damage core was the main factor shaping the initial functional loss and subsequent restoration. On average, the damage locus comprised a circular area $100 \mu \mathrm{m}$ in diameter that accounted for $\sim 3 \%$ of all neurons in the network, which were irreversibly lost. The directly affected regions were those located $\sim 0.7 \mathrm{~mm}$ from damage. Recovery was initiated in regions $\sim 1.5 \mathrm{~mm}$ away, and functional restoration reached the affected regions in $\sim 15 \mathrm{~min}$.

Reorganization of brain circuits after damage involves structural and functional changes that compensate for both the lesion itself and remote effects in the brain (van Meer et al., 2012; Jiang et al., 2013; Grefkes and Fink, 2014; Siegel et al., 2016). In our experiments, however, it is unlikely that structural remodeling of intercluster connectivity is the major mechanism underlying the observed network recovery given the short time scales involved. Although axonal growth and formation of new synaptic connections are fast processes (Marrs et al., 2001; Malyshevskaya et al., 2013), few hours would be required in our preparations to bypass the lost neuronal cluster and rewire the neighboring clusters among themselves, which are typically $1 \mathrm{~mm}$ apart. This time scale of hours is far beyond the 15 min observed for recovery. Thus, we hypothesize that the central role of the physical network after damage is to support the rerouting of intercluster dynamic interactions, reshaping information flow and functional reorganization. On recovery, the major traits of the functional network, namely global efficiency and density of effective links, were similar to the predamage condition. This suggests that the neuronal network not only restored function, but that regulated itself to secure adequate operation levels. Such homeostatic mechanisms have been reported in studies of brains affected by lesions (Butz-Ostendorf and van Ooyen, 2017), and have been hypothesized to play a central role at early stages of recovery.

Despite the differences between in vitro and in vivo systems, our results are in striking accordance with previous studies in mouse brains. Lim and collaborators (Lim et al., 2014) followed network activity with a voltage sensitive dye on optogenetic stimulation of different areas after cortical damage by stroke. They showed that the extent of network affection depended on the connectivity strength between the monitored brain areas and damage locus. Nearby, peri-infarct areas were severely affected, whereas more distant, weakly connected areas remained unaltered. Lim's study also showed that recovery initiated in distant areas and progressed heterogeneously toward the infarcted region. Despite the different size and time scales of Lim's work as compared to ours, the similarity between the studies illustrates the importance of connectivity-based approaches to investigate recovery after local acute damage (Zhu et al., 2010; Grefkes and Fink, 2014). In vitro experiments in combination with network analyses thus provide an excellent platform to relate connectivity failure with functional alterations and recovery mechanisms. The gap between in vivo and in vitro architectures can be reduced through neuroengineering, which allows to mimic major organizational (Aebersold et al., 2016) and dynamical (Yamamoto et al., 2018) features of brain circuits while maintaining full access to neurons and connections. The analysis of damage and recovery in these advanced designs will open new avenues for understanding the link between complex network topologies, damage and functional resilience, more prominently in the context of modular organization (Sporns and Betzel, 2016), and node centrality (Alstott et al., 2009; Fornito et al., 2015).

The mechanisms of recovery observed in our study have important clinical implications. Cheng and coworkers (Cheng et al., 2014) demonstrated that optogenetic stimulation of cortical areas located in the vicinity of a stroke-injured mouse brain promoted overall activity and enhanced multiple plasticity-associated mechanisms, which altogether fostered whole-brain functional restoration. Translated to our in vitro model, such stimulation protocol would correspond to induce activity in the first and second neighborhood rings around the ablated cluster, which would possibly accelerate functional recovery. Our in vitro system is highly tunable and stimulation protocols of different nature, optogenetic, electrical, or chemical, can be easily integrated. In combination with our present capacity of precise surgery and wholenetwork monitoring, stimulation approaches could facilitate a deeper comprehension of the processes underlying network reorganization during recovery, and could foster the development of new therapeutic strategies in affected brains.

To conclude, we emphasize that the restitution of damaged circuitry and overall functional remodeling constitute central mechanisms to prevent a fatal cascade of failures or the complete inoperability of neuronal networks. Our work shows that functional remodeling is fast and robust. Following the recent study of Harush and Barzel (2017), we advocate that dynamic reorganization and the access to diverse pathways for information flow are much more important for resilience than previously thought. Our in vitro approach brings new experimental opportunities and opens new frontiers to comprehend the intricacy of dynamic interactions and functional reorganization in complex networks.

\section{References}

Aebersold MJ, Dermutz H, Forró C, Weydert S, Thompson-Steckel G, Vörös J, Demkó L (2016) "Brains on a chip": towards engineered neural networks. Trends Analyt Chem 78:60-69. 
Allen EA, Damaraju E, Plis SM, Erhardt EB, Eichele T, Calhoun VD (2014) Tracking whole-brain connectivity dynamics in the resting state. Cereb Cortex 24:663-676.

Alstott J, Breakspear M, Hagmann P, Cammoun L, Sporns O (2009) Modeling the impact of lesions in the human brain. PLoS Comput Biol 5:e1000408.

Butz-Ostendorf M, van Ooyen A (2017) Is lesion-induced synaptic rewiring driven by activity homeostasis? In: The rewiring brain (van Ooyen A, Butz-Ostendorf M, eds), pp 71-92. San Diego: Academic Press.

Carmichael ST (2016) The 3 rs of stroke biology: radial, relayed, and regenerative. Neurotherapeutics 13:348-359.

Carrera E, Tononi G (2014) Diaschisis: past, present, future. Brain 137:2408-2422.

Cheng MY, Wang EH, Woodson WJ, Wang S, Sun G, Lee AG, Arac A, Fenno LE, Deisseroth K, Steinberg GK (2014) Optogenetic neuronal stimulation promotes functional recovery after stroke. Proc Natl Acad Sci USA 111:12913-12918.

Corbetta M, Ramsey L, Callejas A, Baldassarre A, Hacker CD, Siegel JS, Astafiev SV, Rengachary J, Zinn K, Lang CE, Connor LT, Fucetola R, Strube M, Carter AR, Shulman GL (2015) Common behavioral clusters and subcortical anatomy in stroke. Neuron 85:927-941.

de Santos-Sierra D, Sendiña-Nadal I, Leyva I, Almendral JA, Anava S, Ayali A, Papo D, Boccaletti S (2014) Emergence of small-world anatomical networks in self-organizing clustered neuronal cultures. PLoS One 9:e85828.

Fornito A, Zalesky A, Breakspear M (2015) The connectomics of brain disorders. Nat Rev Neurosci 16:159.

Grefkes C, Fink GR (2014) Connectivity-based approaches in stroke and recovery of function. Lancet Neurol 13:206-216.

Harush U, Barzel B (2017) Dynamic patterns of information flow in complex networks. Nat Commun 8:2181.

Holloway PM, Gavins FNE (2016) Modeling ischemic stroke in vitro: status quo and future perspectives. Stroke 47:561-569.

Jiang L, Xu H, Yu C (2013) Brain connectivity plasticity in the motor network after ischemic stroke. Neural Plast 2013:924192.

Jones DT, Vemuri P, Murphy MC, Gunter JL, Senjem ML, Machulda MM, Przybelski SA, Gregg BE, Kantarci K, Knopman DS, Boeve BF, Petersen RC, Jack CR Jr (2012) Non-stationarity in the "resting brain's" modular architecture. PLoS One 7:e39731.

Kiviniemi V, Vire T, Remes J, Abou Elseoud A, Starck T, Tervonen O, Nikkinen $\mathrm{J}$ (2011) A sliding time-window ica reveals spatial variability of the default mode network in time. Brain Connect 1:339347.

Lim DH, LeDue JM, Mohajerani MH, Murphy TH (2014) Optogenetic mapping after stroke reveals network-wide scaling of functional connections and heterogeneous recovery of the peri-infarct. $J$ Neurosci 34:16455-16466.

Majdandzic A, Podobnik B, Buldyrev SV, Kenett DY, Havlin S, Eugene Stanley H (2013) Spontaneous recovery in dynamical networks. Nat Phys 10:34-38.

Malyshevskaya O, Shiraishi Y, Kimura F, Yamamoto N (2013) Role of electrical activity in horizontal axon growth in the developing cortex: a time-lapse study using optogenetic stimulation. PLoS One 8:e82954

Marrs GS, Green SH, Dailey ME (2001) Rapid formation and remodeling of postsynaptic densities in developing dendrites. Nat Neurosci 4:1006-1013.

Mathew M, Santos SI, Zalvidea D, Loza-Alvarez P (2009) Multimodal optical workstation for simultaneous linear, nonlinear microscopy and nanomanipulation: upgrading a commercial confocal inverted microscope. Rev Sci Instrum 80:073701.

Murphy TH, Corbett D (2009) Plasticity during stroke recovery: from synapse to behaviour. Nat Rev Neurosci 10:861-872.

Orlandi JG, Soriano J, Alvarez-Lacalle E, Teller S, Casademunt J (2013) Noise focusing and the emergence of coherent activity in neuronal cultures. Nat Phys 9:582-590.

Richard MJP, Saleh TM, El Bahh B, Zidichouski JA (2010) A novel method for inducing focal ischemia in vitro. $J$ Neurosci Methods 190:20-27.

Rubinov M, Sporns O (2010) Complex network measures of brain connectivity: uses and interpretations. Neuroimage 52:10591069.

Sakoğlu U, Pearlson GD, Kiehl KA, Wang YM, Michael AM, Calhoun VD (2010) A method for evaluating dynamic functional network connectivity and task-modulation: application to schizophrenia. MAGMA 23:351-366.

Santos SI, Mathew M, Olarte OE, Psilodimitrakopoulos S, LozaAlvarez P (2013) Femtosecond laser axotomy in Caenorhabditis elegans and collateral damage assessment using a combination of linear and nonlinear imaging techniques. PLoS One 8:e58600.

Segev R, Benveniste M, Shapira Y, Ben-Jacob E (2003) Formation of electrically active clusterized neural networks. Phys Rev Lett 90: 168101.

Siddique R, Thakor N (2014) Investigation of nerve injury through microfluidic devices. J R Soc Interface 11:20130676.

Siegel JS, Ramsey LE, Snyder AZ, Metcalf NV, Chacko RV, Weinberger K, Baldassarre A, Hacker CD, Shulman GL, Corbetta M (2016) Disruptions of network connectivity predict impairment in multiple behavioral domains after stroke. Proc Natl Acad Sci USA 113:E4367-E4376.

Sporns O, Betzel RF (2016) Modular brain networks. Annu Rev Psychol 67:613-640.

Stetter O, Battaglia D, Soriano J, Geisel T (2012) Model-free reconstruction of excitatory neuronal connectivity from calcium imaging signals. PLoS Comput Biol 8:e1002653.

Teller S, Granell C, De Domenico M, Soriano J, Gómez S, Arenas A (2014) Emergence of assortative mixing between clusters of cultured neurons. PLoS Comput Biol 10:e1003796.

Teller S, Tahirbegi IB, Mir M, Samitier J, Soriano J (2015) Magnetiteamyloid- $\beta$ deteriorates activity and functional organization in an in vitro model for Alzheimer's disease. Sci Rep 5:17261.

Thayil AKN, Pereira A, Mathew M, Artigas D, Blanco EM, LozaAlvarez P (2008) Decrease in laser ablation threshold for epithelial tissue microsurgery in a living drosophila embryo during dorsal closure. J Microsc 232:362-368.

van Meer MPA, Otte WM, van der Marel K, Nijboer CH, Kavelaars A, van der Sprenkel JWB, Viergever MA, Dijkhuizen RM (2012) Extent of bilateral neuronal network reorganization and functional recovery in relation to stroke severity. J Neurosci 32:4495-4507.

Vogel A, Noack J, Hüttman G, Paltauf G (2005) Mechanisms of femtosecond laser nanosurgery of cells and tissues. Appl Phys B 81:1015-1047.

Yamamoto H, Moriya S, Ide K, Hayakawa T, Akima H, Sato S, Kubota S, Tanii T, Niwano M, Teller S, Soriano J, Hirano-Iwata A (2018) Impact of modular organization on dynamical richness in cortical networks. Sci Adv 4:eaau4914.

Zhu C, Fan F, He Y, Zang Y, Zhang Y, Wang L, Yu C, Li K, Qin W, Chen H, Wang M, Woodward TS (2010) Dynamic functional reorganization of the motor execution network after stroke. Brain 133:1224-1238 